Article

\title{
Chebyshev-Type Integral Inequalities for Continuous Fields of Operators Concerning Khatri-Rao Products and Synchronous Properties
}

\author{
Arnon Ploymukda and Pattrawut Chansangiam *(D) \\ Department of Mathematics, Faculty of Science, King Mongkut's Institute of Technology Ladkrabang, \\ Bangkok 10520, Thailand; arnon.p.math@gmail.com \\ * Correspondence: pattrawut.ch@kmitl.ac.th; Tel.: +66-935-266600
}

Received: 30 January 2020; Accepted: 1 March 2020; Published: 5 March 2020

check for updates

\begin{abstract}
We consider bounded continuous fields of self-adjoint operators which are parametrized by a locally compact Hausdorff space $\Omega$ equipped with a finite Radon measure $\mu$. Under certain assumptions on synchronous Khatri-Rao property of the fields of operators, we obtain Chebyshev-type inequalities concerning Khatri-Rao products. We also establish Chebyshev-type inequalities involving Khatri-Rao products and weighted Pythagorean means under certain assumptions of synchronous monotone property of the fields of operators. The Pythagorean means considered here are three classical symmetric means: the geometric mean, the arithmetic mean, and the harmonic mean. Moreover, we derive the Chebyshev-Grüss integral inequality via oscillations when $\mu$ is a probability Radon measure. These integral inequalities can be reduced to discrete inequalities by setting $\Omega$ to be a finite space equipped with the counting measure. Our results provide analog results for matrices and integrable functions. Furthermore, our results include the results for tensor products of operators, and Khatri-Rao/Kronecker/Hadamard products of matrices, which have been not investigated in the literature.
\end{abstract}

Keywords: Chebyshev sum inequality; Khatri-Rao product; Bochner integral; weighted Pythagorean mean

MSC: 47A63; 47A64; 47A80

\section{Introduction}

In mathematical analysis and applications, analytic inequalities serve as fundamental tools for comparison, approximation, and optimization. The classical Chebyshev sum inequality states that for any real tuples $\left(a_{1}, \ldots, a_{n}\right)$ and $\left(b_{1}, \ldots, b_{n}\right)$ both are increasing or decreasing, we have

$$
\frac{1}{n} \sum_{i=1}^{n} a_{i} b_{i} \geqslant\left(\frac{1}{n} \sum_{i=1}^{n} a_{i}\right)\left(\frac{1}{n} \sum_{i=1}^{n} b_{i}\right)
$$

This inequality was generalized by Matharu and Aujla [1] to the case of positive semidefinite matrices involving the Hadamard (entrywise) product $\circ$ : for any matrices $A_{1} \geqslant \cdots \geqslant A_{n} \geqslant 0$ and $B_{1} \geqslant \cdots \geqslant B_{n} \geqslant 0$, and any positive numbers $\omega_{1} \ldots, \omega_{n}$, we have

$$
\sum_{i=1}^{n} \omega_{i} \sum_{i=1}^{n} \omega_{i}\left(A_{i} \circ B_{i}\right) \geqslant\left(\sum_{i=1}^{n} \omega_{i} A_{i}\right) \circ\left(\sum_{i=1}^{n} \omega_{i} B_{i}\right) .
$$


To discuss a continuous version of this inequality in a compact form, let us denote for each integrable function $f, g:[a, b] \rightarrow \mathbb{R}$,

$$
\begin{aligned}
\mathcal{I}(f) & =\frac{1}{b-a} \int_{a}^{b} f(x) d x, \\
T_{\mathcal{I}}(f, g) & =\mathcal{I}(f \cdot g)-\mathcal{I}(f) \cdot \mathcal{I}(g) .
\end{aligned}
$$

The latter is called the general Chebyshev functional (see [2]). The Chebyshev functional (4) has many applications in numerical quadrature, probability and statistics, and existence for solutions to certain differential equations. It was obtained in [3] that if such $f$ and $g$ are synchronous on $[a, b]$, that is,

$$
(f(x)-f(y))(g(x)-g(y)) \geqslant 0
$$

for all $x, y \in[a, b]$, then

$$
T_{\mathcal{I}}(f, g) \geqslant 0 .
$$

The opposite inequality of (6) holds if both $f$ and $g$ are opposite-synchronous on $[a, b]$. Operator extensions of this inequality were presented by Moslehian and Bakherad [4]. They generalized the Chebyshev integral inequality (6) to the case of continuous fields of Hilbert space operators with a bounded measurable function involving Hadamard products by using the notion of synchronous Hadamard property. They proved that if two continuous fields $\left(A_{t}\right)_{t \in \Omega},\left(B_{t}\right)_{t \in \Omega}$ of operators, parametrized by a compact Hausdorff space $\Omega$ equipped with a Radon measure $\mu$, have the synchronous Hadamard property, then

$$
\int_{\Omega} \alpha(s) d \mu(s) \int_{\Omega} \alpha(t)\left(A_{t} \odot B_{t}\right) d \mu(t) \geqslant \int_{\Omega} \alpha(t) A_{t} d \mu(t) \odot \int_{\Omega} \alpha(s) B_{s} d \mu(s),
$$

where $\alpha: \Omega \rightarrow[0, \infty)$ is a bounded measurable function. Moreover, they gave some Chebyshev-type inequalities concerning operator means and Hadamard products.

A complement of (6) was introduced by Grüss [5], providing an estimate of the difference between the integral of the product and the product of the integrals for two functions. For any integrable functions $f, g:[a, b] \rightarrow \mathbb{R}$ satisfying the conditions $k \leqslant f(x) \leqslant K, l \leqslant g(x) \leqslant L$ for all $x \in[a, b]$ and $k, K, l, L$ are real constants, we have

$$
\left|T_{\mathcal{I}}(f, g)\right| \leqslant \frac{1}{4}(K-k)(L-l)
$$

We can apply Grüss inequalities to estimate error bounds for some integral means and numerical quadrature rules; see e.g., [6,7]. In [8], Gonska, Raşa and Rusu used the terminology Chebyshev-Grüss inequalities referring to Grüss inequalities for (special cases of) generalized Chebyshev functionals $T_{\mathcal{I}}$ which have a general form

$$
\left|T_{\mathcal{I}}(f, g)\right| \leqslant E(\mathcal{I}, f, g),
$$

where $E$ is an expression in terms of certain properties of $\mathcal{I}$ and some kind of oscillations of $f$ and $g$. They also established new Chebyshev-Grüss inequalities via discrete oscillations.

On the other hand, in the theory of operator product, the notion of tensor product for Hilbert space operators was extended to the Tracy-Singh product for such operators [9]. Algebraic, order, and analytic properties of the Tracy-Singh product for operator were discussed in $[9,10]$. The notion of tensor product was also generalized to the Khatri-Rao product for Hilbert space operators in [11]. 
The work [11] shows that the Khatri-Rao product and the Tracy-Singh product are related via isometric selection operators.

In this paper, we establish new several integral inequalities of Chebyshev-type for continuous fields of self-adjoint operators involving Khatri-Rao products and operator means. In Section 2, we give preliminaries on Khatri-Rao and Tracy-Singh products for operators, and Bochner integrability of continuous field of operators on a locally compact Hausdorff space. In Section 3, we provide Chebyshev-type inequalities involving Khatri-Rao products of operators under the assumption of synchronous Khatri-Rao property. In Section 4, we establish Chebyshev integral inequalities concerning weighted Pythagorean means and Khatri-Rao products under the assumption of synchronous monotone property. We prove Chebyshev-Grüss inequalities via oscillations for continuous fields of operators in Section 5. Our results generalize the matrix result [1] and the result for integrable functions [8]. Moreover, our results include the results for tensor products of operators, and Khatri-Rao/Kronecker/Hadamard products of matrices, which have been not investigated in the literature. Finally, we summarize our work in Section 6.

\section{Preliminaries}

Throughout this paper, let $\mathbb{H}$, and $\mathbb{K}$ be complex Hilbert spaces. The symbol $\mathcal{B}(\mathbb{H})$ stands to the Banach space of bounded linear operators from $\mathbb{H}$ into itself. The vector space of self-adjoint operators on $\mathbb{H}$ is denoted by $\mathcal{B}(\mathbb{H})^{s a}$. Denote the set of all positive (positive invertible, respectively) operators on $\mathbb{H}$ by $\mathcal{B}(\mathbb{H})^{+}\left(\mathcal{B}(\mathbb{H})^{++}\right.$, resp.). For any $A, B \in \mathcal{B}(\mathbb{H})^{s a}$, the situation $A \geqslant B(A>B$, resp.) means that $A-B \in \mathcal{B}(\mathbb{H})^{+}\left(A-B \in \mathcal{B}(\mathbb{H})^{++}\right.$, resp.).

Through this paper, we apply the projection theorem to decompose

$$
\mathbb{H}=\bigoplus_{i=1}^{n} \mathbb{H}_{i}, \quad \mathbb{K}=\bigoplus_{i=1}^{n} \mathbb{K}_{i},
$$

where all $\mathbb{H}_{i}$ and $\mathbb{K}_{i}$ are Hilbert spaces. Each operator $A \in \mathcal{B}(\mathbb{H})$ and $B \in \mathcal{B}(\mathbb{K})$ can be expressed uniquely as operator matrices

$$
A=\left[A_{i j}\right]_{i, j=1}^{n, n} \text { and } B=\left[B_{i j}\right]_{i, j=1}^{n, n},
$$

where $A_{i j} \in \mathbb{B}\left(\mathbb{H}_{j}, \mathbb{H}_{i}\right)$ and $B_{i j} \in \mathbb{B}\left(\mathbb{K}_{j}, \mathbb{K}_{i}\right)$ for each $i, j$.

\subsection{Khatri-Rao Product and Tracy-Singh Product for Operators}

Recall that the tensor product of $A \in \mathcal{B}(\mathbb{H})$ and $B \in \mathcal{B}(\mathbb{K})$ is a unique bounded linear operator $A \otimes B \in \mathcal{B}(\mathbb{H} \otimes \mathbb{K})$ such that for all $x \in \mathbb{H}$ and all $y \in \mathbb{K}$,

$$
(A \otimes B)(x \otimes y)=A x \otimes B y .
$$

Fix a countable orthonormal basis $\mathbb{E}$ of $\mathbb{H}$. Recall that the Hadamard product of $A, B \in \mathcal{B}(\mathbb{H})$ is defined to be the operator $A \odot B \in \mathcal{B}(\mathbb{H})$ such that for all $e \in \mathbb{E}$,

$$
\langle(A \odot B) e, e\rangle=\langle A e, e\rangle\langle B e, e\rangle .
$$

It is known that the Hadamard product of $A, B \in \mathcal{B}(\mathbb{H})$ can be expressed as

$$
A \odot B=U^{*}(A \otimes B) U,
$$

where $U: \mathbb{H} \rightarrow \mathbb{H} \otimes \mathbb{H}$ is the isometry defined by $U e=e \otimes e$ for all $e \in \mathbb{E}$ (see e.g., [12]). 
From the previous setting, we define the Khatri-Rao product of $A$ and $B$ to be the bounded linear operator from $\bigoplus_{i=1}^{n} \mathbb{H}_{i} \otimes \mathbb{K}_{i}$ into itself represented by an operator matrix

$$
A \triangleright B=\left[A_{i j} \otimes B_{i j}\right]_{i j} .
$$

We define the Tracy-Singh product of $A$ and $B$ to be the bounded linear operator from $\bigoplus_{i=1}^{n} \bigoplus_{j=1}^{n} \mathbb{H}_{i} \otimes \mathbb{K}_{j}$ into itself represented by an operator matrix

$$
A \otimes B=\left[\left[A_{i j} \otimes B_{k l}\right]_{k l}\right]_{i j} .
$$

The maps $(A, B) \mapsto A \boxminus B$ and $(A, B) \mapsto A \otimes B$ are bilinear. Moreover, we have:

Lemma 1 ([9-11]). Let $A, B, C, D$ be compatible operators. Then

1. If $A \geqslant C \geqslant 0$ and $B \geqslant D \geqslant 0$, then $A \boxminus B \geqslant C \square D \geqslant 0$.

2. If $A \geqslant C \geqslant 0$ and $B \geqslant D \geqslant 0$, then $A \otimes B \geqslant C \otimes D \geqslant 0$.

3. $(A \otimes B)(C \otimes D)=(A C) \otimes(B D)$.

4. $(A+C) \otimes(B+D)=A \otimes B+A \otimes D+C \otimes B+C \otimes D$.

5. If $A$ and $B$ are invertible, then $(A \otimes B)^{-1}=A^{-1} \otimes B^{-1}$.

6. If $A$ and $B$ are positive, then $(A \otimes B)^{\alpha}=A^{\alpha} \otimes B^{\alpha}$ for any $\alpha \geqslant 0$.

Lemma 2 ([11]). There is an isometry $Z$ such that $A \boxminus B=Z^{*}(A \otimes B) Z$ for any operators $A \in \mathcal{B}(\mathbb{H})$ and $B \in \mathcal{B}(\mathbb{K})$.

\subsection{Bochner Integration for Operator-Valued Maps}

Throughout this paper, let $\Omega$ be a locally compact Hausdorff space endowed with a finite Radon measure $\mu$. A continuous map $A: \Omega \rightarrow \mathbb{X} \subseteq \mathcal{B}(\mathbb{H})$ is called a continuous field of operators in $\mathbb{X}$ parametrized by $\Omega$, denoted by $A \in \mathfrak{C}(\Omega ; \mathbb{X})$. For convenience, for each $t \in \Omega$, we may write $A_{t}$ instead of $A(t)$. The field $A$ is said to be bounded if there is a constant $M>0$ such that $\left\|A_{t}\right\| \leqslant M$ for all $t \in \Omega$. The set of all bounded continuous fields of operators in $\mathbb{X}$ parametrized by $\Omega$ is denoted by $\mathfrak{B C}(\Omega ; \mathbb{X})$. If $A \in \mathfrak{C}(\Omega ; \mathbb{X})$ is such that the norm function $t \mapsto\left\|A_{t}\right\|$ is Lebergue integrable on $\Omega$ (e.g., $A \in \mathfrak{B C}(\Omega ; \mathbb{X}))$, then we can form the Bochner integral $\int_{\Omega} A_{t} d \mu(t)$.

Lemma 3 (see e.g., [13]). Let $\mathbb{X}$ and $\mathbb{Y}$ be Banach spaces, and $\varphi: \mathbb{X} \rightarrow \mathbb{Y}$ a bounded linear operator. For any Bochner integrable function $f: \Gamma \rightarrow \mathbb{X}$, the composition $\varphi \circ f$ is also Bochner integrable and

$$
\int_{\Gamma}(\varphi \circ f) d v=\varphi \int_{\Gamma} f d v
$$

Proposition 1. For any $A \in \mathfrak{B C}(\Omega, \mathcal{B}(\mathbb{H}))$ and $X \in \mathcal{B}(\mathbb{K})$, we have

$$
\int_{\Omega} A_{t} d \mu(t) \triangleright X=\int_{\Omega}\left(A_{t} \boxminus X\right) d \mu(t) .
$$

Proof. The map $t \mapsto A_{t}$ is Bochner integrable on $\Omega$ because it is continuous. Since the map $T \mapsto T \boxminus X$ is bounded linear operator, we have by Lemma 3 that the map $t \mapsto A_{t} \boxminus X$ is Bochner integrable on $\Omega$ and (13) holds.

\section{Chebyshev-Type Inequalities Involving Khatri-Rao Products of Operators}

We introduce the following property, and prove Chebyshev-type inequalities involving Khatri-Rao products of operators. 
Definition 1. The fields $A$ and $B$ of operators parametrized by $\Omega$ are said to have the synchronous Khatri-Rao property if, for all $s, t \in \Omega$,

$$
\left(A_{t}-A_{s}\right) \triangleright\left(B_{t}-B_{s}\right) \geqslant 0 .
$$

They are said to have the opposite-synchronous Khatri-Rao property if the reverse of (14) holds for all $s, t \in \Omega$.

The following result is an extension of the Chebyshev integral inequality (6) to the case of operators involving Khatri-Rao products.

Theorem 1. Let $A \in \mathfrak{B C}\left(\Omega, \mathcal{B}(\mathbb{H})^{s a}\right)$ and $B \in \mathfrak{B C}\left(\Omega, \mathcal{B}(\mathbb{K})^{s a}\right)$, and let $\alpha: \Omega \rightarrow[0, \infty)$ be a bounded measurable function.

1. If $A$ and $B$ have the synchronous Khatri-Rao property, then

$$
\int_{\Omega} \alpha(s) d \mu(s) \int_{\Omega} \alpha(t)\left(A_{t} \sqsubset B_{t}\right) d \mu(t) \geqslant \int_{\Omega} \alpha(t) A_{t} d \mu(t) \odot \int_{\Omega} \alpha(s) B_{s} d \mu(s) .
$$

2. If $A$ and B have the opposite-synchronous Khatri-Rao property, then then the reverse of (15) holds.

Proof. By using Lemma 1 and Proposition 1, we have

$$
\begin{aligned}
& \int_{\Omega} \alpha(s) d \mu(s) \int_{\Omega} \alpha(t)\left(A_{t} \boxminus B_{t}\right) d \mu(t)-\int_{\Omega} \alpha(t) A_{t} d \mu(t) \triangleright \int_{\Omega} \alpha(s) B_{s} d \mu(s) \\
& =\iint_{\Omega^{2}} \alpha(s) \alpha(t)\left(A_{t} \boxminus B_{t}\right) d \mu(t) d \mu(s)-\iint_{\Omega^{2}} \alpha(t) \alpha(s)\left(A_{t} \boxminus B_{s}\right) d \mu(t) d \mu(s) \\
& =\iint_{\Omega^{2}}\left[\alpha(s) \alpha(t)\left(A_{t} \boxminus B_{t}\right)-\alpha(t) \alpha(s)\left(A_{t} \boxminus B_{s}\right)\right] d \mu(t) d \mu(s) \\
& =\frac{1}{2} \iint_{\Omega^{2}}\left[\alpha(s) \alpha(t)\left(A_{t} \boxminus B_{t}\right)-\alpha(t) \alpha(s)\left(A_{t} \boxminus B_{s}\right)\right] d \mu(t) d \mu(s) \\
& +\frac{1}{2} \iint_{\Omega^{2}}\left[\alpha(t) \alpha(s)\left(A_{s} \sqcup B_{s}\right)-\alpha(s) \alpha(t)\left(A_{s} \sqcup B_{t}\right)\right] d \mu(s) d \mu(t) \\
& =\frac{1}{2} \iint_{\Omega^{2}} \alpha(s) \alpha(t)\left[\left(A_{t}-A_{s}\right) \triangleright\left(B_{t}-B_{s}\right)\right] d \mu(t) d \mu(s) .
\end{aligned}
$$

Here, we have used Fubini's Theorem [14] to interchange the order of integrals. For the case 1, we have

$$
\iint_{\Omega^{2}} \alpha(s) \alpha(t)\left[\left(A_{t}-A_{s}\right) \triangleright\left(B_{t}-B_{s}\right)\right] d \mu(t) d \mu(s) \geqslant 0
$$

and thus (15) holds. For another case, we get the reverse of (16) and, thus, the reverse of (15) holds.

For the case $n=1$, i.e., $\mathbb{H}$ and $\mathbb{K}$ are not decomposed, the synchronous Khatri-Rao property in Definition 1 reduces to the synchronous tensor property:

$$
\left(A_{t}-A_{s}\right) \otimes\left(B_{t}-B_{s}\right) \geqslant 0 .
$$

If two fields $A$ and $B$ of operators parametrized by $\Omega$ have the synchronous tensor property, then $A$ and $B$ have the synchronous Hadamard property ([4], Definition 2.1), i.e.,

$$
\left(A_{t}-A_{s}\right) \odot\left(B_{t}-B_{s}\right) \geqslant 0
$$

for all $s, t \in \Omega$. The following result gives Chebyshev-type inequalities involving tensor products and Hadamard products. 
Corollary 1. Let $A, B \in \mathfrak{B C}\left(\Omega, \mathcal{B}(\mathbb{H})^{s a}\right)$ and let $\alpha: \Omega \rightarrow[0, \infty)$ be a bounded measurable function.

1. If $A$ and $B$ have the synchronous tensor property, then

$$
\begin{aligned}
& \int_{\Omega} \alpha(s) d \mu(s) \int_{\Omega} \alpha(t)\left(A_{t} \otimes B_{t}\right) d \mu(t) \geqslant \int_{\Omega} \alpha(t) A_{t} d \mu(t) \otimes \int_{\Omega} \alpha(s) B_{s} d \mu(s), \\
& \int_{\Omega} \alpha(s) d \mu(s) \int_{\Omega} \alpha(t)\left(A_{t} \odot B_{t}\right) d \mu(t) \geqslant \int_{\Omega} \alpha(t) A_{t} d \mu(t) \odot \int_{\Omega} \alpha(s) B_{s} d \mu(s) .
\end{aligned}
$$

2. If $A$ and $B$ have the opposite-synchronous tensor property, then then the reverses of (17) and (18) holds.

Proof. For the case $n=1$, the Khatri-Rao product in Theorem 1 reduces to the tensor product. Assume that $A$ and $B$ have the synchronous tensor property. Using the fact that the Hadamard product is expressed as the deformation of tensor product via the isometry $U$ defined in (10), we obtain

$$
\begin{aligned}
\int_{\Omega} \alpha(s) d \mu(s) \int_{\Omega} \alpha(t)\left(A_{t} \odot B_{t}\right) d \mu(t) & =\int_{\Omega} \alpha(s) d \mu(s) \int_{\Omega} \alpha(t) U^{*}\left(A_{t} \otimes B_{t}\right) U d \mu(t) \\
& =U^{*}\left(\int_{\Omega} \alpha(s) d \mu(s) \int_{\Omega} \alpha(t)\left(A_{t} \otimes B_{t}\right) d \mu(t)\right) U \\
& \geqslant U^{*}\left(\int_{\Omega} \alpha(t) A_{t} d \mu(t) \otimes \int_{\Omega} \alpha(s) B_{s} d \mu(s)\right) U \\
& =\int_{\Omega} \alpha(t) A_{t} d \mu(t) \odot \int_{\Omega} \alpha(s) B_{s} d \mu(s) .
\end{aligned}
$$

Case 2 for Hadamard products can be similarly treated.

We can see that the inequality (18) is the same as (7), but they hold under different hypothesis. The next corollary is a discrete version of Theorem 1.

Corollary 2. Let $A=\left(A_{1}, \ldots, A_{k}\right)$ and $B=\left(B_{1}, \ldots, B_{k}\right)$ where $A_{i} \in \mathcal{B}(\mathbb{H})^{s a}, B_{i} \in \mathcal{B}(\mathbb{K})^{s a}$ and $\omega_{i}$ is a nonnegative number for each $i=1, \ldots, k$.

1. If $A$ and $B$ have the synchronous Khatri-Rao property, then

$$
\sum_{i=1}^{k} \omega_{i} \sum_{i=1}^{k} \omega_{i}\left(A_{i} \boxminus B_{i}\right) \geqslant\left(\sum_{i=1}^{k} \omega_{i} A_{i}\right) \square\left(\sum_{i=1}^{k} \omega_{i} B_{i}\right) .
$$

2. If $A$ and $B$ have the opposite-synchronous Khatri-Rao property, then the reverse of (19) holds.

Proof. From the previous theorem, consider the finite space $\Omega=\{1, \ldots, k\}$ equipped with the counting measure and $\alpha(i)=\omega_{i}$ for all $i=1, \ldots, k$.

This corollary generalizes Chebyshev sum inequalities for the case of real numbers in inequality (1) and for Hadamard product of matrices in [1].

Next, we illustrate Chebyshev-type inequalities for bounded linear operators induced from matrices. Recall that with each $A \in \mathbb{M}_{n}(\mathbb{C})$ one can naturally associate a bounded linear operator

$$
T_{A}: \mathbb{C}^{n} \rightarrow \mathbb{C}^{n}, \quad x \mapsto A x
$$

For any complex matrices $A=\left[A_{i j}\right]$ and $B=\left[B_{i j}\right]$ partitioned in block-matrix form, we have [11]

$$
T_{A} \bullet T_{B}=T_{A \triangleright B} .
$$


Example 1. Consider $\omega_{1}=\omega_{2}=\frac{1}{2}, A=\left(T_{A_{1}}, T_{A_{2}}\right)$, and $B=\left(T_{B_{1}}, T_{B_{2}}\right)$, where

$$
\begin{aligned}
& A_{1}=\left[\begin{array}{c|cc}
-1 & 1 & -1 \\
\hline 1 & 2 & 0 \\
-1 & 0 & -3
\end{array}\right], \quad A_{2}=\left[\begin{array}{c|cc}
2 & 1 & 0 \\
\hline 1 & 3 & -1 \\
0 & -1 & 1
\end{array}\right], \\
& B_{1}=\left[\begin{array}{cc|c}
1 & 1 & -1 \\
1 & -1 & 0 \\
\hline-1 & 0 & 1
\end{array}\right], \quad B_{2}=\left[\begin{array}{cc|c}
2 & 1 & 0 \\
1 & 4 & 0 \\
\hline 0 & 0 & 2
\end{array}\right] .
\end{aligned}
$$

First, we check the hypothesis of Corollary 2. Since $A_{2}-A_{1} \geqslant 0$, we have $T_{A_{2}}-T_{A_{1}} \geqslant 0$. Similarly, $T_{B_{2}}-T_{B_{1}} \geqslant 0$. By the positivity of the Khatri-Rao product, we get

$$
\left(T_{A_{2}}-T_{A_{1}}\right) \bullet\left(T_{B_{2}}-T_{B_{1}}\right) \geqslant 0,
$$

i.e., the fields $A=\left(T_{A_{1}}, T_{A_{2}}\right)$ and $B=\left(T_{B_{1}}, T_{B_{2}}\right)$ have the synchronous Khatri-Rao product property.

Now, we can check that the following matrix is positive semidefinite:

$$
\begin{aligned}
A_{1} \triangleright B_{1}+A_{2} \triangleright B_{2} & -\frac{1}{2}\left(A_{1}+A_{2}\right) \square\left(B_{1}+B_{2}\right) \\
& =\left[\begin{array}{cc|cc}
3 & 1 & -1 & 1 \\
1 & 9 & 0 & 0 \\
\hline-1 & 0 & 8 & -2 \\
1 & 0 & -2 & -1
\end{array}\right]-\frac{1}{2}\left[\begin{array}{cc|cc}
3 & 2 & -2 & 1 \\
2 & 3 & 0 & 0 \\
\hline-2 & 0 & 15 & -3 \\
1 & 0 & -3 & -6
\end{array}\right] \\
& =\frac{1}{2}\left[\begin{array}{cc|cc}
3 & 0 & 0 & 1 \\
0 & 15 & 0 & 0 \\
\hline 0 & 0 & 1 & -1 \\
1 & 0 & -1 & 4
\end{array}\right] \\
& \geqslant 0 .
\end{aligned}
$$

Thus,

$$
A_{1} \boxminus B_{1}+A_{2} \boxminus B_{2} \geqslant \frac{1}{2}\left(A_{1}+A_{2}\right) \boxminus\left(B_{1}+B_{2}\right) .
$$

Passing through the induced linear maps, we get

$$
T_{A_{1} \boxminus B_{1}+A_{2} \boxminus B_{2}} \geqslant T_{\frac{1}{2}\left(A_{1}+A_{2}\right) \boxminus\left(B_{1}+B_{2}\right)} .
$$

Finally, applying the property (20), we have

$$
T_{A_{1}} \square T_{B_{1}}+T_{A_{2}} \square T_{B_{2}} \geqslant \frac{1}{2}\left(T_{A_{1}}+T_{A_{2}}\right) \square\left(T_{B_{1}}+T_{B_{2}}\right),
$$

i.e., the inequality (19) in Corollary 2 holds.

\section{Chebyshev Integral Inequalities Concerning Weighted Pythagorean Means of Operators}

We start this section by introducing order assumptions on continuous fields and supplying preliminaries on weighted arithmetic/geometric/harmonic means of operators. The main part is to establish Chebyshev-type inequalities involving Khatri-Rao products concerning such operator means and order assumptions.

Throughout this section, the space $\Omega$ is equipped with a total ordering $\preccurlyeq$. Consider the following definitions: 
Definition 2. We say that a field $A$ is increasing (decreasing, resp.) whenever $s \preccurlyeq t$ implies $A_{s} \leqslant A_{t}$ $\left(A_{s} \geqslant A_{t}\right.$, respectivley).

Definition 3. Two ordered pairs $\left(X_{1}, X_{2}\right)$ and $\left(Y_{1}, Y_{2}\right)$ of self-adjoint operators are said to have the synchronous property if either

$$
X_{i} \leqslant Y_{i} \text { for } i=1,2 \text {, or } X_{i} \geqslant Y_{i} \text { for } i=1,2 \text {. }
$$

The pairs $\left(X_{1}, X_{2}\right)$ and $\left(Y_{1}, Y_{2}\right)$ are said to have the opposite-synchronous property if either

$$
X_{1} \leqslant Y_{1} \text { and } X_{2} \geqslant Y_{2} \text {, or } X_{1} \geqslant Y_{1} \text { and } X_{2} \leqslant Y_{2} \text {. }
$$

Definition 4. Let $A, B, C, D$ be continuous fields of self-adjoint operators parametrized by $\Omega$. Two ordered pairs $(A, B)$ and $(C, D)$ are said to have the synchronous monotone property if $\left(A_{t}, B_{t}\right)$ and $\left(C_{t}, D_{t}\right)$ have the synchronous property for all $t \in \Omega$. The pairs $(A, B)$ and $(C, D)$ are said to have the opposite-synchronous monotone property if $\left(A_{t}, B_{t}\right)$ and $\left(C_{t}, D_{t}\right)$ have the opposite-synchronous property for all $t \in \Omega$.

Recall that the three classical Pythagorean means are the following symmetric means: the arithmetic mean, the harmonic mean, and the geometric mean. For each $w \in[0,1]$, the $w$-weighted versions of such means are respectively defined for any $A, B \in \mathcal{B}(\mathbb{H})^{++}$by

$$
\begin{aligned}
& A \nabla_{w} B=(1-w) A+w B, \\
& A !_{w} B=\left[(1-w) A^{-1}+w B^{-1}\right]^{-1}, \\
& A \sharp_{w} B=A^{\frac{1}{2}}\left(A^{-\frac{1}{2}} B A^{-\frac{1}{2}}\right)^{w} A^{\frac{1}{2}} .
\end{aligned}
$$

These means can be defined for arbitrary positive operators by the following continuity argument with respect to the strong-operator topology:

$$
A \sharp_{w} B=\lim _{\varepsilon \rightarrow 0^{+}}(A+\varepsilon I) \sharp_{w}(B+\varepsilon I) .
$$

For brevity, we write $A \sharp B$ for $A \sharp_{1 / 2} B$. The Pythagorean means have the following remarkable property: for any $w \in[0,1], T \in \mathcal{B}(\mathbb{H})$, and $A, B \in \mathcal{B}(\mathbb{H})^{+}$, we have

$$
T^{*}\left(A \sigma_{w} B\right) T \leqslant\left(T^{*} A T\right) \sigma_{w}\left(T^{*} B T\right),
$$

here $\sigma$ is anyone of $\nabla, !, \#$.

Lemma 4 (see e.g., [15]). The weighted geometric means, weighted arithmetic means and weighted harmonic means for operators are (jointly) monotone in the sense that if $X_{1} \leqslant X_{2}$ and $Y_{1} \leqslant Y_{2}$, then $X_{1} \sigma Y_{1} \leqslant X_{2} \sigma Y_{2}$ where $\sigma$ is any of $\nabla_{w}, !_{w}, \sharp w_{w}$.

\subsection{Inequalities on Weighted Geometric Means}

Recall that a linear map between two operator algebras is said to be positive if it maps positive operators to positive operators.

Lemma 5 ([16]). Let $A, B, C, D \in \mathcal{B}(\mathbb{H})^{+}$and $w \in[0,1]$. Then

$$
(A \boxminus B) \sharp_{w}(C \boxminus D) \geqslant\left(A \sharp_{w} C\right) \square\left(B \sharp_{w} D\right) .
$$


Theorem 2. Let $A, B, C, D \in \mathfrak{B C}\left(\Omega ; \mathcal{B}(\mathbb{H})^{+}\right)$and let $\alpha: \Omega \rightarrow[0, \infty)$ be a bounded measurable function. If $A, B, C, D$ are either all increasing, or all decreasing, then

$$
\int_{\Omega} \alpha(s) d \mu(s) \int_{\Omega} \alpha(t)\left[\left(A_{t} \boxminus B_{t}\right) \sharp_{w}\left(C_{t} \square D_{t}\right)\right] d \mu(t) \geqslant \int_{\Omega} \alpha(t)\left(A_{t} \sharp_{w} C_{t}\right) d \mu(t) \square \int_{\Omega} \alpha(s)\left(B_{s} \sharp_{w} D_{s}\right) d \mu(s) .
$$

Proof. Let $s, t \in \Omega$. Without loss of generality, assume that $s \preccurlyeq t$. By applying Lemmas 1 and 5 , Proposition 1, and Fubini's Theorem [14], we have

$$
\begin{aligned}
& \int_{\Omega} \alpha(s) d \mu(s) \int_{\Omega} \alpha(t)\left[\left(A_{t} \boxminus B_{t}\right) \sharp_{w}\left(C_{t} \boxminus D_{t}\right)\right] d \mu(t)-\int_{\Omega} \alpha(t)\left(A_{t} \sharp_{w} C_{t}\right) d \mu(t) \triangleright \int_{\Omega} \alpha(s)\left(B_{s} \sharp_{w} D_{s}\right) d \mu(s) \\
& =\iint_{\Omega^{2}} \alpha(s) \alpha(t)\left[\left(A_{t} \odot B_{t}\right) \sharp_{w}\left(C_{t} \odot D_{t}\right)\right] d \mu(t) d \mu(s)-\iint_{\Omega^{2}} \alpha(t) \alpha(s)\left[\left(A_{t} \sharp_{w} C_{t}\right) \odot\left(B_{s} \sharp_{w} D_{s}\right)\right] d \mu(t) d \mu(s) \\
& \geqslant \iint_{\Omega^{2}} \alpha(s) \alpha(t)\left[\left(A_{t} \sharp_{w} C_{t}\right) \sqcup\left(B_{t} \sharp_{w} D_{t}\right)\right] d \mu(t) d \mu(s)-\iint_{\Omega^{2}} \alpha(t) \alpha(s)\left[\left(A_{t} \sharp_{w} C_{t}\right) \sqcup\left(B_{s} \sharp_{w} D_{s}\right)\right] d \mu(t) d \mu(s) \\
& =\iint_{\Omega^{2}} \alpha(s) \alpha(t)\left[\left(A_{t} \sharp_{w} C_{t}\right) \odot\left(B_{t} \sharp_{w} D_{t}\right)-\left(A_{t} \sharp_{w} C_{t}\right) \triangleright\left(B_{s} \sharp_{w} D_{s}\right)\right] d \mu(t) d \mu(s) \\
& =\frac{1}{2} \iint_{\Omega^{2}} \alpha(s) \alpha(t)\left[\left(A_{t} \sharp_{w} C_{t}\right) \sqcup\left(B_{t} \sharp_{w} D_{t}\right)-\left(A_{t} \sharp_{w} C_{t}\right) \sqcup\left(B_{s} \sharp_{w} D_{s}\right)\right] d \mu(t) d \mu(s) \\
& +\frac{1}{2} \iint_{\Omega^{2}} \alpha(t) \alpha(s)\left[\left(A_{s} \sharp_{w} C_{s}\right) \square\left(B_{s} \sharp_{w} D_{s}\right)-\left(A_{s} \sharp_{w} C_{s}\right) \sqcup\left(B_{t} \sharp_{w} D_{t}\right)\right] d \mu(s) d \mu(t) \\
& =\frac{1}{2} \iint_{\Omega^{2}} \alpha(s) \alpha(t)\left[A_{t} \sharp_{w} C_{t}-A_{s} \sharp_{w} C_{s}\right] \bullet\left[B_{t} \sharp_{w} D_{t}-B_{s} \sharp_{w} D_{s}\right] d \mu(t) d \mu(s) \text {. }
\end{aligned}
$$

If $A, B, C, D$ are all increasing, we have by Lemma 4 that $A_{t} \sharp_{w} C_{t} \geqslant A_{s} \sharp_{w} C_{s}$ and $B_{t} \sharp_{w} D_{t} \geqslant B_{s} \sharp_{w} D_{s}$. If $A, B, C, D$ are all decreasing, we have $A_{t} \sharp_{w} C_{t} \leqslant A_{s} \sharp_{w} C_{s}$ and $B_{t} \sharp_{w} D_{t} \leqslant B_{s} \sharp_{w} D_{s}$. By Lemma 1 , both cases lead to the same conclusion that $\left(A_{t} \sharp_{w} C_{t}-A_{s} \sharp_{w} C_{s}\right) \sqcup\left(B_{t} \sharp_{w} D_{t}-B_{s} \sharp_{w} D_{s}\right) \geqslant 0$, and hence (22) holds.

The next corollary is a discrete version of Theorem 1.

Corollary 3. Let $A=\left(A_{1}, \ldots, A_{k}\right), B=\left(B_{1}, \ldots, B_{k}\right), C=\left(C_{1}, \ldots, C_{k}\right)$ and $D=\left(D_{1}, \ldots, D_{k}\right)$ where $A_{i}, B_{i}, C_{i}, D_{i} \in \mathcal{B}(\mathbb{H})^{+}$for each $i=1, \ldots, k$. If $A, B, C, D$ are either all increasing, or all decreasing, then

$$
\frac{1}{k} \sum_{i=1}^{k}\left[\left(A_{i} \boxminus B_{i}\right) \sharp_{w}\left(C_{i} \boxminus D_{i}\right)\right] \geqslant\left(\frac{1}{k} \sum_{i=1}^{k}\left(A_{i} \sharp_{w} C_{i}\right)\right) \unrhd\left(\frac{1}{k} \sum_{i=1}^{k}\left(B_{i} \sharp_{w} D_{i}\right)\right) .
$$

Proof. Setting $\Omega=\{1, \ldots, k\}$ equipped with the counting measure and $\alpha(i)=\frac{1}{k}$ for all $i=1, \ldots, k$ in Theorem 2, we get the result.

Operator inequality (23) can be regarded as a generalization of the Chebyshev sum inequality (1). The next goal is to establish a reverse version of Theorem 2 .

Lemma 6. Let $A, B, C, D \in \mathcal{B}(\mathbb{H})^{+}$be such that $0<m_{1} I \leqslant A \otimes B \leqslant M_{1} I$ and $0<m_{2} I \leqslant C \otimes D \leqslant M_{2} I$. Denote $m=\frac{m_{2}}{M_{1}}, M=\frac{M_{2}}{m_{1}}$ and

$$
\lambda=\frac{M m^{w}-m M^{w}}{(1-w)(M-m)} \cdot\left(\frac{1-w}{w} \cdot \frac{M^{w}-m^{w}}{M m^{w}-m M^{w}}\right)^{w}
$$

Then for any $w \in(0,1)$, we have

$$
\lambda(A \odot B) \sharp_{w}(C \sqcup D) \leqslant\left(A \sharp_{w} C\right) \triangleright\left(B \sharp_{w} D\right) .
$$


Proof. Consider a map $\Phi: T \mapsto Z^{*} T Z$, where $Z$ is the isometry in Lemma 2. Since $\Phi$ is a unital positive linear map, we have by ([17], Corollary 3.5) that

$$
\lambda\left[\Phi(A \otimes B) \#_{w} \Phi(C \otimes D)\right] \leqslant \Phi\left((A \otimes B) \#_{w}(C \otimes D)\right) .
$$

From ([16], Theorem 1), we get

$$
(A \otimes B) \#_{w}(C \otimes D)=\left(A \#_{w} C\right) \otimes\left(B \#_{w} D\right) .
$$

Hence,

$$
\begin{aligned}
\lambda\left[(A \boxminus B) \#_{w}(C \boxminus D)\right] & =\lambda\left[\Phi(A \otimes B) \#_{w} \Phi(C \otimes D)\right] \\
& \leqslant \Phi\left((A \otimes B) \#_{w}(C \otimes D)\right) \\
& =\Phi\left(\left(A \#_{w} C\right) \bigotimes\left(B \#_{w} D\right)\right) \\
& =\left(A \#_{w} C\right) \square\left(B \#_{w} D\right) .
\end{aligned}
$$

Theorem 3. Let $A, B, C, D \in \mathfrak{B C}\left(\Omega ; \mathcal{B}(\mathbb{H})^{+}\right)$with $0<m_{1} I \leqslant A_{t} \otimes B_{t} \leqslant M_{1} I$ and $0<m_{2} I \leqslant C_{t} \otimes D_{t} \leqslant$ $M_{2}$ I for all $t \in \Omega$, and $\alpha: \Omega \rightarrow[0, \infty)$ a bounded measurable function. Let $m, M, w, \lambda$ as in Lemma 6 . If either

1. $A, C$ are increasing and $B, D$ are decreasing, or

2. $A, C$ are decreasing and $B, D$ are increasing,

then

$$
\begin{aligned}
\lambda \int_{\Omega} \alpha(s) d \mu(s) \int_{\Omega} \alpha(t)\left[\left(A_{t} \boxminus B_{t}\right) \sharp_{w}\left(C_{t} \triangleright D_{t}\right)\right] d \mu(t) & \\
& \leqslant \int_{\Omega} \alpha(t)\left(A_{t} \sharp_{w} C_{t}\right) d \mu(t) \triangleright \int_{\Omega} \alpha(s)\left(B_{s} \sharp_{w} D_{s}\right) d \mu(s) .
\end{aligned}
$$

Proof. Let $s, t \in \Omega$. Without loss of generality, assume that $s \preccurlyeq t$. By applying Lemmas 1 and 6 , Proposition 1, and Fubini's Theorem [14], we have

$$
\begin{aligned}
& \lambda \int_{\Omega} \alpha(s) d \mu(s) \int_{\Omega} \alpha(t)\left[\left(A_{t} \boxminus B_{t}\right) \sharp_{w}\left(C_{t} \boxminus D_{t}\right)\right] d \mu(t)-\int_{\Omega} \alpha(t)\left(A_{t} \sharp_{w} C_{t}\right) d \mu(t) \unrhd \int_{\Omega} \alpha(s)\left(B_{s} \sharp_{w} D_{s}\right) d \mu(s) \\
& =\iint_{\Omega^{2}} \alpha(s) \alpha(t)\left[\lambda\left(A_{t} \boxminus B_{t}\right) \sharp_{w}\left(C_{t} \boxminus D_{t}\right)\right] d \mu(t) d \mu(s) \\
& -\iint_{\Omega^{2}} \alpha(t) \alpha(s)\left[\left(A_{t} \sharp_{w} C_{t}\right) \bullet\left(B_{s} \sharp_{w} D_{s}\right)\right] d \mu(t) d \mu(s) \\
& \leqslant \iint_{\Omega^{2}} \alpha(s) \alpha(t)\left[\left(A_{t} \sharp_{w} C_{t}\right) \odot\left(B_{t} \sharp_{w} D_{t}\right)\right] d \mu(t) d \mu(s) \\
& -\iint_{\Omega^{2}} \alpha(t) \alpha(s)\left[\left(A_{t} \sharp_{w} C_{t}\right) \triangleright\left(B_{s} \sharp_{w} D_{s}\right)\right] d \mu(t) d \mu(s) \\
& =\frac{1}{2} \iint_{\Omega^{2}} \alpha(s) \alpha(t)\left[\left(A_{t} \sharp_{w} C_{t}\right) \sqcup\left(B_{t} \sharp_{w} D_{t}\right)-\left(A_{t} \sharp_{w} C_{t}\right) \triangleright\left(B_{s} \sharp_{w} D_{s}\right)\right] d \mu(t) d \mu(s) \\
& +\frac{1}{2} \iint_{\Omega^{2}} \alpha(t) \alpha(s)\left[\left(A_{s} \sharp_{w} C_{s}\right) \sqcup\left(B_{s} \sharp_{w} D_{s}\right)-\left(A_{s} \sharp_{w} C_{s}\right) \sqcup\left(B_{t} \sharp_{w} D_{t}\right)\right] d \mu(s) d \mu(t) \\
& =\frac{1}{2} \iint_{\Omega^{2}} \alpha(s) \alpha(t)\left[A_{t} \sharp_{w} C_{t}-A_{s} \sharp_{w} C_{s}\right] \sqcup\left[B_{t} \sharp_{w} D_{t}-B_{s} \sharp_{w} D_{s}\right] d \mu(t) d \mu(s) \text {. }
\end{aligned}
$$

We have by Lemmas 1 and 4 that $\left(A_{t} \sharp_{w} C_{t}-A_{s} \sharp_{w} C_{s}\right) \odot\left(B_{t} \sharp_{w} D_{t}-B_{s} \sharp_{w} D_{s}\right) \leqslant 0$, and hence (25) holds. 


\subsection{Inequalities on Weighted Arithmetic Means}

Lemma 7 ([18]). Let $A, B, C, D \in \mathcal{B}(\mathbb{H})^{s a}$ and $w \in[0,1]$.

1. If $(A, B)$ and $(C, D)$ have the synchronous property, then

$$
(A \boxminus B) \nabla_{w}(C \boxminus D) \geqslant\left(A \nabla_{w} C\right) \bullet\left(B \nabla_{w} D\right) .
$$

2. If $(A, B)$ and $(C, D)$ have the opposite-synchronous property, then the reverse of (26) holds.

Theorem 4. Let $A, B, C, D \in \mathfrak{B C}\left(\Omega ; \mathcal{B}(\mathbb{H})^{+}\right)$and let $\alpha: \Omega \rightarrow[0, \infty)$ be a bounded measurable function.

1. If $(A, B),(C, D)$ have the synchronous monotone property and all of $A, B, C, D$ are either increasing or decreasing, then

$$
\begin{aligned}
\int_{\Omega} \alpha(s) d \mu(s) \int_{\Omega} \alpha(t)[ & \left.\left(A_{t} \boxminus B_{t}\right) \nabla_{w}\left(C_{t} \boxminus D_{t}\right)\right] d \mu(t) \\
& \geqslant \int_{\Omega} \alpha(t)\left(A_{t} \nabla_{w} C_{t}\right) d \mu(t) \square \int_{\Omega} \alpha(s)\left(B_{s} \nabla_{w} D_{s}\right) d \mu(s) .
\end{aligned}
$$

2. If $(A, B),(C, D)$ have the opposite-synchronous monotone property and if either

(a) $A, C$ are increasing and $B, D$ are decreasing, or

(b) $A, C$ are decreasing and $B, D$ are increasing,

then the reverse of (27) holds.

Proof. Let $s, t \in \Omega$. Without loss of generality, assume that $s \preccurlyeq t$. First, we consider the case 1 . We have by using Lemmas 1 and 7, proposition 1, and Fubini's Theorem that

$$
\begin{aligned}
\int_{\Omega} \alpha(s) d \mu(s) & \int_{\Omega} \alpha(t)\left[\left(A_{t} \boxminus B_{t}\right) \nabla_{w}\left(C_{t} \boxminus D_{t}\right)\right] d \mu(t)-\int_{\Omega} \alpha(t)\left(A_{t} \nabla_{w} C_{t}\right) d \mu(t) \square \int_{\Omega} \alpha(s)\left(B_{s} \nabla_{w} D_{s}\right) d \mu(s) \\
= & \iint_{\Omega^{2}} \alpha(s) \alpha(t)\left[\left(A_{t} \boxminus B_{t}\right) \nabla_{w}\left(C_{t} \boxminus D_{t}\right)\right] d \mu(t) d \mu(s) \\
& -\iint_{\Omega^{2}} \alpha(t) \alpha(s)\left[\left(A_{t} \nabla_{w} C_{t}\right) \square\left(B_{s} \nabla_{w} D_{s}\right)\right] d \mu(t) d \mu(s) \\
\geqslant & \iint_{\Omega^{2}} \alpha(s) \alpha(t)\left[\left(A_{t} \nabla_{w} C_{t}\right) \square\left(B_{t} \nabla_{w} D_{t}\right)\right] d \mu(t) d \mu(s) \\
& -\iint_{\Omega^{2}} \alpha(t) \alpha(s)\left[\left(A_{t} \nabla_{w} C_{t}\right) \square\left(B_{s} \nabla_{w} D_{s}\right)\right] d \mu(t) d \mu(s) \\
= & \iint_{\Omega^{2}} \alpha(s) \alpha(t)\left[\left(A_{t} \nabla_{w} C_{t}\right) \square\left(B_{t} \nabla_{w} D_{t}\right)-\left(A_{t} \nabla_{w} C_{t}\right) \square\left(B_{s} \nabla_{w} D_{s}\right)\right] d \mu(t) d \mu(s) \\
= & \frac{1}{2} \iint_{\Omega^{2}} \alpha(s) \alpha(t)\left[A_{t} \nabla_{w} C_{t}-A_{s} \nabla_{w} C_{s}\right] \square\left[B_{t} \nabla_{w} D_{t}-B_{s} \nabla_{w} D_{s}\right] d \mu(t) d \mu(s) .
\end{aligned}
$$

By Lemmas 1 and 4 , we have $\left(A_{t} \nabla_{w} C_{t}-A_{s} \nabla_{w} C_{s}\right) \square\left(B_{t} \nabla_{w} D_{t}-B_{s} \nabla_{w} D_{s}\right) \geqslant 0$ and hence (27) holds. The case 2 can be similarly treated.

We can illustrate Theorem 4 for the case of operators induced from matrices as follows. 
Example 2. Consider the following pairs of induced bounded linear operators: $A=\left(T_{A_{1}}, T_{A_{2}}\right), B=\left(T_{B_{1}}, T_{B_{2}}\right)$, $C=\left(T_{C_{1}}, T_{C_{2}}\right)$ and $D=\left(T_{D_{1}}, T_{D_{2}}\right)$, where

$$
\begin{aligned}
& A_{1}=\left[\begin{array}{l|ll}
2 & 1 & 0 \\
\hline 1 & 1 & 0 \\
0 & 0 & 2
\end{array}\right], \quad A_{2}=\left[\begin{array}{l|ll}
3 & 1 & 0 \\
\hline 1 & 2 & 1 \\
0 & 1 & 4
\end{array}\right], \quad B_{1}=\left[\begin{array}{l|ll}
3 & 2 & 0 \\
\hline 2 & 2 & 0 \\
0 & 0 & 1
\end{array}\right], \\
& B_{2}=\left[\begin{array}{l|ll}
5 & 3 & 0 \\
\hline 3 & 6 & 2 \\
0 & 2 & 4
\end{array}\right], \quad C_{1}=\left[\begin{array}{l|ll}
2 & 1 & 0 \\
\hline 1 & 1 & 0 \\
0 & 0 & 1
\end{array}\right], \quad C_{2}=\left[\begin{array}{l|ll}
3 & 1 & 0 \\
\hline 1 & 2 & 1 \\
0 & 1 & 1
\end{array}\right] \text {, } \\
& D_{1}=\left[\begin{array}{l|ll}
1 & 1 & 0 \\
\hline 1 & 2 & 0 \\
0 & 0 & 1
\end{array}\right], \quad D_{2}=\left[\begin{array}{l|ll}
1 & 1 & 0 \\
\hline 1 & 3 & 2 \\
0 & 2 & 2
\end{array}\right] \text {. }
\end{aligned}
$$

We can check the hypothesis of Theorem 4: (i) all of $A, B, C, D$ are increasing, and (ii) $(A, B),(C, D)$ have the synchronous monotone property. Set $\Omega=\{1,2\}$ equipped with the counting measure and $\alpha(i)=\frac{1}{2}$ for all $i=1,2$. Let us denote $\nabla=\nabla_{1 / 2}$. Now, a direct computation reveals that

$$
\begin{aligned}
& \left(A_{1} \sqcup B_{1}\right) \nabla\left(C_{1} \square D_{1}\right)+\left(A_{2} \square B_{2}\right) \nabla\left(C_{2} \square D_{2}\right)-\frac{1}{2}\left(A_{1} \nabla C_{1}+A_{2} \nabla C_{2}\right) \square\left(B_{1} \nabla D_{1}+B_{2} \nabla D_{2}\right) \\
& =\frac{1}{4}\left[\begin{array}{c|cccc}
2 & 0 & 0 & 0 & 0 \\
\hline 0 & 6 & 2 & 5 & 2 \\
0 & 2 & 4 & 2 & 4 \\
0 & 5 & 2 & 14 & 8 \\
0 & 2 & 4 & 8 & 10
\end{array}\right] \geqslant 0,
\end{aligned}
$$

or equivalently,

$$
\left(A_{1} \boxminus B_{1}\right) \nabla\left(C_{1} \square D_{1}\right)+\left(A_{2} \square B_{2}\right) \nabla\left(C_{2} \square D_{2}\right) \geqslant \frac{1}{2}\left(A_{1} \nabla C_{1}+A_{2} \nabla C_{2}\right) \square\left(B_{1} \nabla D_{1}+B_{2} \nabla D_{2}\right) .
$$

Passing through the induced linear maps and applying the properties $T_{A \nabla B}=T_{A} \nabla T_{B}$ and (20), we obtain

$$
\begin{aligned}
\left(T_{A_{1}} \triangleright T_{B_{1}}\right) \nabla\left(T_{C_{1}} \triangleright T_{D_{1}}\right) & +\left(T_{A_{2}} \triangleright T_{B_{2}}\right) \nabla\left(T_{C_{2}} \triangleright T_{D_{2}}\right) \\
& \geqslant \frac{1}{2}\left(T_{A_{1}} \nabla T_{C_{1}}+T_{A_{2}} \nabla T_{C_{2}}\right) \square\left(T_{B_{1}} \nabla T_{D_{1}}+T_{B_{2}} \nabla T_{D_{2}}\right) .
\end{aligned}
$$

Thus, the equality (27) holds in this case.

\subsection{Inequalities on Weighted Harmonic Means}

Lemma 8 ([18]). Let $A, B, C, D \in \mathcal{B}(\mathbb{H})^{+}$and $w \in[0,1]$. If $(A, B)$ and $(C, D)$ have the opposite-synchronous property, then

$$
(A \boxminus B) !_{w}(C \sqcup D) \geqslant\left(A !_{w} C\right) \square\left(B !_{w} D\right) .
$$

Theorem 5. Let $A, B, C, D \in \mathfrak{B C}\left(\Omega ; \mathcal{B}(\mathbb{H})^{+}\right)$and $\alpha: \Omega \rightarrow[0, \infty)$ a bounded measurable function. If $(A, B)$ and $(C, D)$ have the opposite-synchronous monotone property and all of $A, B, C, D$ are either increasing or decreasing, then

$$
\begin{aligned}
& \int_{\Omega} \alpha(s) d \mu(s) \int_{\Omega} \alpha(t)\left[\left(A_{t} \boxminus B_{t}\right) !_{w}\left(C_{t} \triangleright D_{t}\right)\right] d \mu(t) \\
& \geqslant \int_{\Omega} \alpha(t)\left(A_{t} !_{w} C_{t}\right) d \mu(t) \triangleright \int_{\Omega} \alpha(s)\left(B_{s} !_{w} D_{s}\right) d \mu(s) .
\end{aligned}
$$


Proof. Let $s, t \in \Omega$ with $s \preccurlyeq t$. By applying Lemmas 1 and 8 , Proposition 1 , and Fubini's theorem, we get

$$
\begin{aligned}
& \int_{\Omega} \alpha(s) d \mu(s) \int_{\Omega} \alpha(t)\left[\left(A_{t} \triangleright B_{t}\right) !_{w}\left(C_{t} \sqcup D_{t}\right)\right] d \mu(t)-\int_{\Omega} \alpha(t)\left(A_{t} !_{w} C_{t}\right) d \mu(t) \triangleright \int_{\Omega} \alpha(s)\left(B_{s} !_{w} D_{s}\right) d \mu(s) \\
& =\iint_{\Omega^{2}} \alpha(s) \alpha(t)\left[\left(A_{t} \boxminus B_{t}\right) !_{w}\left(C_{t} \boxminus D_{t}\right)\right] d \mu(t) d \mu(s) \\
& -\iint_{\Omega^{2}} \alpha(t) \alpha(s)\left[\left(A_{t} !_{w} C_{t}\right) \triangleright\left(B_{s} !_{w} D_{s}\right)\right] d \mu(t) d \mu(s) \\
& \geqslant \iint_{\Omega^{2}} \alpha(s) \alpha(t)\left[\left(A_{t} !_{w} C_{t}\right) \odot\left(B_{t} !_{w} D_{t}\right)\right] d \mu(t) d \mu(s) \\
& -\iint_{\Omega^{2}} \alpha(t) \alpha(s)\left[\left(A_{t} !_{w} C_{t}\right) \sqcup\left(B_{s} !_{w} D_{s}\right)\right] d \mu(t) d \mu(s) \\
& =\frac{1}{2} \iint_{\Omega^{2}} \alpha(s) \alpha(t)\left[A_{t} !_{w} C_{t}-A_{s} !_{w} C_{s}\right] \sqcup\left[B_{t} !_{w} D_{t}-B_{s} !_{w} D_{s}\right] d \mu(t) d \mu(s) .
\end{aligned}
$$

By Lemmas 1 and $4,\left(A_{t} !_{w} C_{t}-A_{s} !_{w} C_{s}\right) \sqcup\left(B_{t} !_{w} D_{t}-B_{s} !_{w} D_{s}\right) \geqslant 0$ and hence (28) holds.

Lemma 9 ([18]). Let $A, B, C, D \in \mathcal{B}(\mathbb{H})^{+}$with $0<m_{1} I \leqslant A \otimes B \leqslant M_{1} I$ and $0<m_{2} I \leqslant C \otimes D \leqslant$ $M_{2}$ I. Denote

$$
\lambda=\left[\frac{\left(m_{1} \sharp M_{1}\right) \nabla_{1-w}\left(m_{2} \sharp M_{2}\right)}{\left(m_{1} \nabla_{1-w} M_{2}\right) \sharp\left(M_{1} \nabla_{1-w} m_{2}\right)}\right]^{2} .
$$

If $(A, B)$ and $(C, D)$ have the synchronous property, then for any $w \in[0,1]$,

$$
\lambda(A \boxminus B) !_{w}(C \sqcup D) \leqslant\left(A !_{w} C\right) \triangleright\left(B !_{w} D\right) .
$$

Theorem 6. Let $A, B, C, D \in \mathfrak{B C}\left(\Omega ; \mathcal{B}(\mathbb{H})^{+}\right)$be such that for all $t \in \Omega, 0<m_{1} I \leqslant A_{t} \otimes B_{t} \leqslant M_{1} I$ and $0<m_{2} I \leqslant C_{t} \otimes D_{t} \leqslant M_{2} I$. Let $\alpha: \Omega \rightarrow[0, \infty)$ be a bounded measurable function. If $(A, B)$ and $(C, D)$ have the synchronous monotone property and if either

1. $A, C$ are increasing and $B, D$ are decreasing, or

2. $A, C$ are decreasing and $B, D$ are increasing,

then with the constant $\lambda$ in (29) we have

$$
\begin{aligned}
\lambda \int_{\Omega} \alpha(s) d \mu(s) & \int_{\Omega} \alpha(t)\left[\left(A_{t} \boxminus B_{t}\right) !_{w}\left(C_{t} \boxminus D_{t}\right)\right] d \mu(t) \\
& \leqslant \int_{\Omega} \alpha(t)\left(A_{t} !_{w} C_{t}\right) d \mu(t) \square \int_{\Omega} \alpha(s)\left(B_{s} !_{w} D_{s}\right) d \mu(s) .
\end{aligned}
$$

Proof. Let $s, t \in \Omega$ with $s \preccurlyeq t$. We have by using Lemmas 1 and 9, Proposition 1, and Fubini's Theorem that

$$
\begin{aligned}
& \lambda \int_{\Omega} \alpha(s) d \mu(s) \int_{\Omega} \alpha(t)\left[\left(A_{t} \triangleright B_{t}\right) !_{w}\left(C_{t} \triangleright D_{t}\right)\right] d \mu(t)-\int_{\Omega} \alpha(t)\left(A_{t} !_{w} C_{t}\right) d \mu(t) \triangleright \int_{\Omega} \alpha(s)\left(B_{s} !_{w} D_{s}\right) d \mu(s) \\
& =\iint_{\Omega^{2}} \alpha(s) \alpha(t)\left[\lambda\left(A_{t} \boxminus B_{t}\right) !_{w}\left(C_{t} \boxminus D_{t}\right)\right] d \mu(t) d \mu(s) \\
& -\iint_{\Omega^{2}} \alpha(t) \alpha(s)\left[\left(A_{t} !_{w} C_{t}\right) \triangleright\left(B_{s} !_{w} D_{s}\right)\right] d \mu(t) d \mu(s) \\
& \leqslant \iint_{\Omega^{2}} \alpha(s) \alpha(t)\left[\left(A_{t} !_{w} C_{t}\right) \sqcup\left(B_{t} !_{w} D_{t}\right)\right] d \mu(t) d \mu(s) \\
& -\iint_{\Omega^{2}} \alpha(t) \alpha(s)\left[\left(A_{t} !_{w} C_{t}\right) \sqcup\left(B_{s} !_{w} D_{s}\right)\right] d \mu(t) d \mu(s) \\
& =\frac{1}{2} \iint_{\Omega^{2}} \alpha(s) \alpha(t)\left[A_{t} !_{w} C_{t}-A_{s} !_{w} C_{s}\right] \boxminus\left[B_{t} !_{w} D_{t}-B_{s} !_{w} D_{s}\right] d \mu(t) d \mu(s) .
\end{aligned}
$$


We have, by Lemmas 1 and $4,\left(A_{t} !_{w} C_{t}-A_{s} !_{w} C_{s}\right) \square\left(B_{t} !_{w} D_{t}-B_{s} !_{w} D_{s}\right) \leqslant 0$ and hence (30) holds.

Remark 1. When we set $\Omega=\{1, \ldots, k\}$ equipped with the counting measure, we get discrete versions of Theorems 3-6. Matrix analogs of our results can be obtained particularly by setting $\mathbb{H}=\mathbb{C}^{n}$. In this case, our results include Chebyshev-type inequalities for Khatri-Rao products, Kronecker products and Hadamard products of matrices.

\section{Chebyshev-Grüss Inequalities via Oscillations}

In this section, we prove a Chebyshev-Grüss inequality via oscillations for continuous fields of operators. The basic setup is as follows.

Let $\Omega$ be a compact Hausdorff space equipped with a probability Radon measure $\mu$. For any $A \in \mathfrak{C}(\Omega ; \mathcal{B}(\mathbb{H}))$ and $B \in \mathfrak{C}(\Omega ; \mathcal{B}(\mathbb{K}))$, we define

$$
\begin{aligned}
A \boxminus B & =\left(A_{t} \square B_{t}\right)_{t \in \Omega,} \\
\mathcal{I}(A) & =\int_{\Omega} A_{t} d \mu(t), \\
\operatorname{osc}(A) & =\max \left\{\left\|A_{t}-A_{s}\right\|:(t, s) \in \operatorname{supp}(\mu \times \mu)\right\} .
\end{aligned}
$$

Here, we recall that the support of the product measure $\mu \times \mu$ is defined by

$$
\operatorname{supp}(\mu \times \mu)=\left\{(t, s) \in \Omega^{2} \mid(\mu \times \mu)(G)>0 \text { for all open sets } G \subseteq \Omega^{2} \text { containing }(t, s)\right\}
$$

We call $\operatorname{osc}(A)$ the oscillation of the field $A$.

In the next theorem, we generalize Grüss inequality (8) to the case of operators concerning Khatri-Rao products.

Theorem 7. For any $A \in \mathfrak{C}\left(\Omega ; \mathcal{B}(\mathbb{H})^{s a}\right)$ and $B \in \mathfrak{C}\left(\Omega ; \mathcal{B}(\mathbb{K})^{s a}\right)$, we have

$$
\mathcal{I}(A \boxminus B)-\mathcal{I}(A) \bullet \mathcal{I}(B) \leqslant \frac{1}{2} \operatorname{osc}(A) \operatorname{osc}(B)(\mu \times \mu)\left(\Omega^{2} \backslash \Delta\right) I,
$$

where $\Delta=\{(t, t): t \in \Omega\}$.

Proof. By using Lemma 1, Proposition 1 and Fubini's theorem, we have

$$
\begin{aligned}
\mathcal{I}(A \boxminus B)-\mathcal{I}(A) \triangleright \mathcal{I}(B) & =\int_{\Omega} d \mu(s) \int_{\Omega} A_{t} \boxminus B_{t} d \mu(t)-\int_{\Omega} A_{t} d \mu(t) \square \int_{\Omega} B_{s} d \mu(s) \\
& =\iint_{\Omega^{2}} A_{t} \boxminus B_{t} d \mu(t) d \mu(s)-\iint_{\Omega^{2}} A_{t} \boxminus B_{s} d \mu(t) d \mu(s) \\
& =\frac{1}{2} \iint_{\Omega^{2}}\left(A_{t}-A_{s}\right) \triangleright\left(B_{t}-B_{s}\right) d \mu(t) d \mu(s) \\
& =\frac{1}{2} \iint_{\Omega^{2} \backslash \Delta}\left(A_{t}-A_{s}\right) \square\left(B_{t}-B_{s}\right) d \mu(t) d \mu(s) \\
& \leqslant \frac{1}{2} \operatorname{osc}(A) \operatorname{osc}(B)(\mu \times \mu)\left(\Omega^{2} \backslash \Delta\right) I .
\end{aligned}
$$

Theorem 7 can be viewed as an operator version of ([8], Theorem 7).

Corollary 4. For any $A_{i} \in \mathcal{B}(\mathbb{H})^{s a}$ and $B_{i} \in \mathcal{B}(\mathbb{K})^{s a}, i=1, \ldots, k$, we have

$$
\sum_{i=1}^{k}\left(A_{i} \boxminus B_{i}\right)-\left(\sum_{i=1}^{k} A_{i}\right) \sqcup\left(\sum_{i=1}^{k} B_{i}\right) \leqslant \frac{k(k-1)}{2} \max _{1 \leqslant i, j \leqslant k}\left\|A_{i}-A_{j}\right\| \cdot \max _{1 \leqslant i, j \leqslant k}\left\|B_{i}-B_{j}\right\| I .
$$


Proof. Let $A=\left(A_{1}, \ldots, A_{k}\right)$ and $B=\left(B_{1} \ldots, B_{k}\right)$. Set $\Omega$ to be the finite space $\{1, \ldots, k\}$ equipped with the counting measure in Theorem 7 , we have

$$
(\mu \times \mu)\left(\Omega^{2} \backslash \Delta\right)=\frac{k(k-1)}{2}, \quad \operatorname{supp}(\mu \times \mu)=\Omega^{2}
$$

and thus $\operatorname{osc}(A)=\max _{1 \leqslant i, j \leqslant k}\left\|A_{i}-A_{j}\right\|, \operatorname{osc}(B)=\max _{1 \leqslant i, j \leqslant k}\left\|B_{i}-B_{j}\right\|$.

\section{Conclusions and Future Work}

We investigate integral inequalities of Chebyshev-type for bounded continuous fields of self-adjoint operators which are parametrized by a locally compact Hausdorff space $\Omega$ equipped with a finite Radon measure $\mu$. Under certain assumptions on the synchronous Khatri-Rao property of the fields of operators, we obtain Chebyshev-type inequalities concerning Khatri-Rao products. We also establish Chebyshev-type inequalities involving Khatri-Rao products and weighted Pythagorean means under the assumption of synchronous monotone property of the fields of operators. Moreover, we derive Chebyshev-Grüss integral inequality via oscillations when $\mu$ is a probability Radon measure. These integral inequalities can be reduced to discrete inequalities by setting $\Omega$ to be a finite space equipped with the counting measure. Our results include Chebyshev-type inequalities for the tensor product of operators and Khatri-Rao/Kronecker/Hadamard products of matrices.

For future work, we may investigate Chebyshev-type inequalities when replacing weighted Pythagorean means by quasi-arithmetic power means, the logarithmic mean, or general operator means.

Author Contributions: All authors contributed equally and significantly in writing this article. All authors have read and agreed to the published version of the manuscript.

Funding: The first author expresses his gratitude towards Thailand Science Research and Innovation (Thailand Research Fund) for providing the Royal Golden Jubilee Ph.D. Scholarship, grant no. PHD60K0225 to support his Ph.D. study.

Acknowledgments: This work was supported by Thailand Science Research and Innovation (Thailand Research Fund).

Conflicts of Interest: The authors declare no conflict of interest.

\section{References}

1. Matharu, J.S.; Aujla, J.S. Hadamard product versions of the Chebyshev and Kantorovich inequalities. J. Inequal. Pure Appl. Math. 2009, 10, 51.

2. Chebyshev, P.L. Sur les expressions approximatives des intégrales définies par les autres prises entre les mêmes limites. Proc. Math. Soc. Kharkov 1882, 2, 93-98. Translated in Oeuvres 1907, 2, 716-719. (In Russian)

3. Chebyshev, P.L. O priblizhennyh vyrazhenijah odnih integralov cherez drugie. Soobschenija i Protokoly Zasedanij Mathematicheskogo Obschestva pri Imperatorskom. Khar'kovskom Universitete 1882, 2, 93-98.

4. Moslehian, M.S.; Bakherad, M. Chebyshev type inequalities for Hilbert space operators. J. Math. Anal. Appl. 2014, 420, 737-749. [CrossRef]

5. Grüss, G. Über das Maximum des absoluten Betrages von $\frac{1}{b-a} \int_{a}^{b} f(x) g(x) d x-\frac{1}{(b-a)^{2}} \int_{a}^{b} f(x) d x \cdot \int_{a}^{b} g(x) d x$. Mathematische Zeitschrift 1935, 39, 215-226. [CrossRef]

6. Dragomir, S.S.; Wang, S. An inequality of Ostrowski-Grüss type and its applications to the estimation of error bounds for some means and for some numerical quadrature rules. Comput. Math. Appl. 1997, 33, 15-20. [CrossRef]

7. Fedotov, I.; Dragomir, S.S. An inequality of Ostrowski type and its applications for Simpson's rule and special means. Math. Inequal. Appl. 1999, 2, 491-499. [CrossRef]

8. Gonska, H.; Raşa, I.; Rusu, M.D. Chebyshev-Grüss-type inequalities via discrete oscillations. Buletinul Academiei de Stiinte a Republicii Moldova. Matematica 2014, 74, 63-89.

9. Ploymukda, A.; Chansangiam, P.; Lewkeeratiyutkul, W. Algebraic and order properties of Tracy-Singh products for operator matrices. J. Comput. Anal. Appl. 2018, 24, 656-664. 
10. Ploymukda, A.; Chansangiam, P.; Lewkeeratiyutkul, W. Analytic properties of Tracy-Singh products for operator matrices. J. Comput. Anal. Appl. 2018, 24, 665-674.

11. Ploymukda, A.; Chansangiam, P. Khatri-Rao products of operator matrices acting on the direct sum of Hilbert spaces. J. Math. 2016, 2016. [CrossRef]

12. Fujii, J.I. The Marcus-Khan theorem for Hilbert space operators, Mathematica Japonica 1995, 41, 531-535.

13. Aliprantis, C.D.; Border, K.C. Infinite Dimensional Analysis: A Hitchhiker's Guide; Springer: New York, NY, USA, 2006.

14. Bogdanowicz, W.M. Fubini theorems for generalized Lebesgue-Bochner-Stieltjes integral. Proc. Jpn. Acad. 1966, 41, 979-983. [CrossRef]

15. Kubo, F.; Ando, T. Means of positive linear operators. Math. Ann. 1980, 246, 205-224. [CrossRef]

16. Ploymukda, A.; Chansangiam, P. Geometric means and Tracy-Singh products for positive operators. Commun. Math. Appl. 2018, 9, 475-488.

17. Mićić, J.; Pečarić, J.; Seo, Y. Complementary inequalities to inequalities of Jensen and Ando based on the Mond-Pečarić method. Linear Algebra Appl. 2000, 318, 87-107. [CrossRef]

18. Ploymukda, A.; Chansangiam, P. Inequalities on weighted classical Pythagorean means, Tracy-Singh products, and Khatri-Rao products for Hermitian operators. Malays. J. Fundam. Appl. Sci. 2020, 16, to appear.

(C) 2020 by the authors. Licensee MDPI, Basel, Switzerland. This article is an open access article distributed under the terms and conditions of the Creative Commons Attribution (CC BY) license (http://creativecommons.org/licenses/by/4.0/). 